\title{
Effect of morphine, prostigmine, pethidine, and probanthine on the human colon in diverticulosis studied by intraluminal pressure recording and cineradiography
}

\author{
NEIL STAMFORD PAINTER, S. C. TRUELOVE, G. M. ARDRAN, AND M. TUCKEY \\ From the Nuffield Department of Clinical Medicine and the Nuffield Institute for Medical \\ Research, University of Oxford, Oxford
}

EDITORIAL SYNOPSIS A previous study has shown that morphine causes the generation of high intrasigmoid pressures. In diverticulosis of the colon, this effect is exaggerated in those segments actually bearing diverticula. There is therefore a prima facie case for questioning the wisdom of giving morphine to patients with diverticulosis. In the present investigation, cineradiography was used in conjunction with intraluminal pressure recording to study the behaviour of colonic diverticula after the administration of morphine and other drugs. High intrasigmoid pressures evoked by morphine are shown to be accompanied by distension of neighbouring diverticula, sometimes to an extreme degree. Although the necks of the diverticula may shut under the influence of morphine, they do not remain closed but open intermittently and allow colonic contents to be squirted into the diverticula. It appears possible that the use of morphine in acute diverticulitis may predispose to rupture of inflamed diverticula. These effects of morphine can be abolished by an intravenous injection of the anticholinergic agent, probanthine. The analgesic drug, pethidine (demerol), does not have the undesirable effects of morphine and it is suggested that this drug is to be preferred to morphine for the relief of pain in acute diverticulitis.

The administration of morphine to patients with diverticulosis results in the generation of an excessive number of waves of high intraluminal pressure in the part of the sigmoid colon that bears diverticula; by contrast, the unaffected parts of the sigmoid produce pressure patterns essentially similar to those of the normal sigmoid colon (Painter and Truelove, 1964a). A similar but not identical response of the diseased sigmoid is evoked by prostigmine (neostigmine methylsulphate) (Painter and Truelove, 1964b). Both these drugs cause the sigmoid colon to segment, and this segmentation appears to have an intimate connexion with the capacity of the colon to generate localized intraluminal pressures (Painter, Truelove, Ardran, and Tuckey, 1964). An injection of probanthine (propantheline bromide) abolishes the high pressures that are evoked by morphine and prostigmine (Painter and Truelove, 1964c). The analgesic agent pethidine (demerol) differs from morphine in causing a diminution of both the number and the dimensions of the intraluminal waves of pressure (Painter and Truelove, 1964c).
The effect of morphine raises a question which is of immediate practical importance. High intrasigmoid pressures are potentially dangerous in acute diverticulitis as they might cause inflamed diverticula to rupture. However, it could be argued that morphine might cause the colonic musculature to contract in such a way as to occlude the necks of the diverticula and thus isolate the diverticula from the force of the intracolonic pressures. We have therefore employed cineradiography in combination with intraluminal pressure recording to study the behaviour of the colon in diverticulosis when it is being influenced by morphine or by other drugs.

\section{METHOD}

The method of investigation and the apparatus used have already been described (Painter, 1962). The number of subjects studied is given in Table $I$.

\section{RESULTS}

Figure 1 consists of a pressure tracing together with 
TABLE I

NUMBER OF PATIENTS STUDIED WITH COMBINED CINERADIOGRAPHY AND INTRALUMINAL PRESSURE RECORDING

\begin{tabular}{lcc} 
Drug Administered & \multicolumn{2}{c}{ Number of Patients } \\
\cline { 2 - 3 } & Normal & Diverticulosis \\
\hline Morphine & 7 & 19 \\
Pethidine & 1 & 5 \\
Prostigmine & 5 & 9 \\
Probanthine & 4 & 4 \\
Total & 17 & 37
\end{tabular}

frames from the corresponding cinefilm. The exposure of each frame of this film is indicated at the top of the pressure tracing by a series of notches; every tenth notch is numbered. The frames were exposed at the rate of one per second. The subject was a woman of 58 with a single diverticulum in the sigmoid colon which had already been filmed under basal conditions (Painter et al., 1964). The present film was taken after she had been given morphine. The tip of a recording lead (lead 2) can be seen in the immediate vicinity of the diverticulum at the top of the print near the number 2. Much of the barium has collected in the upper sigmoid leaving the bowel near the diverticulum less full of barium. This distribution of the barium is the result of a contraction of the sigmoid with partial occlusion of the colonic lumen in the vicinity of the diverticulum. During this film, lead 2, which was adjacent to the diverticulum, recorded two waves of pressure which

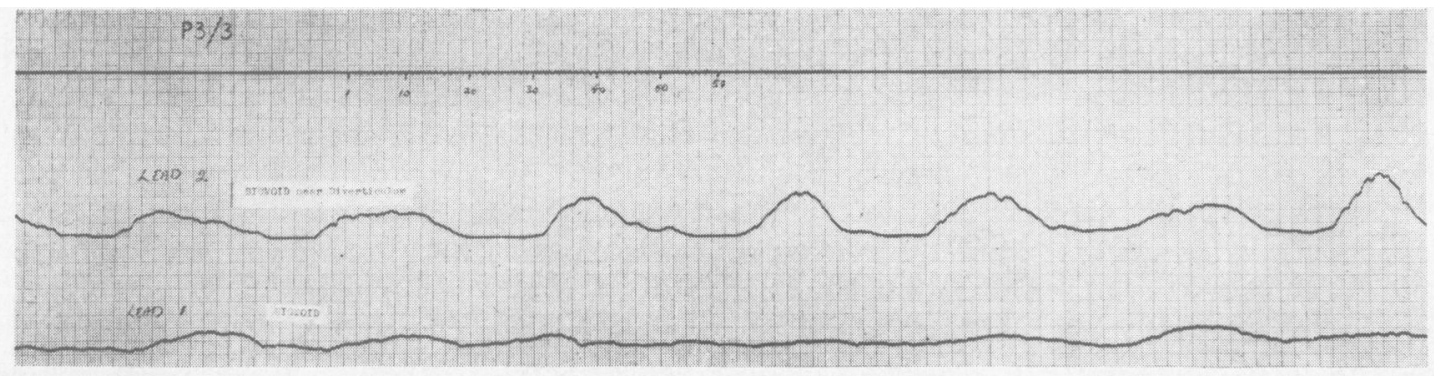

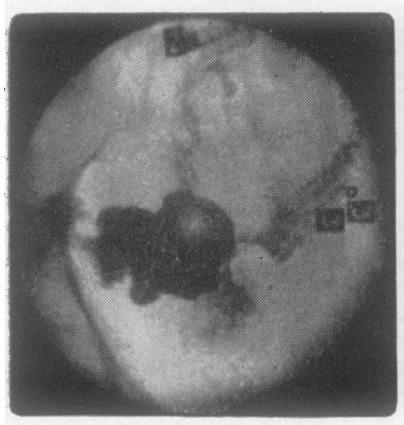

Frame 10

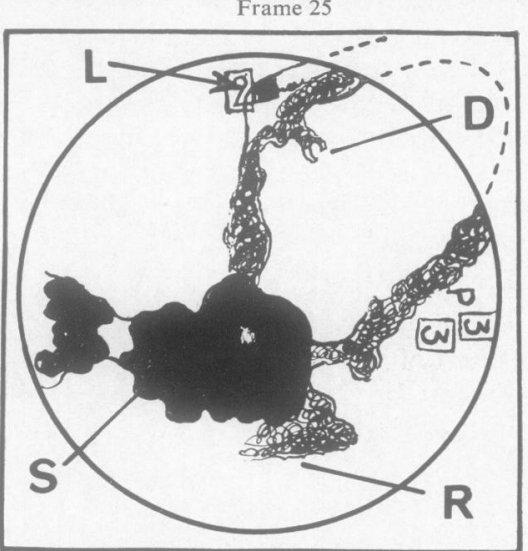

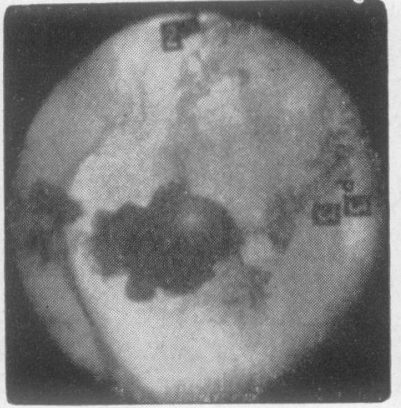

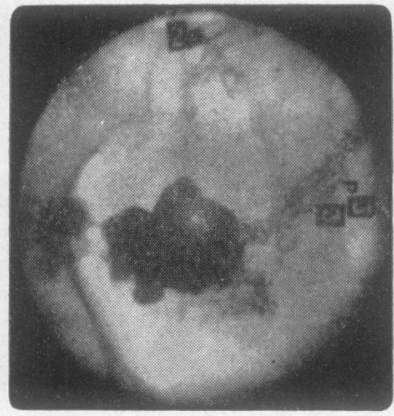

Frame 38

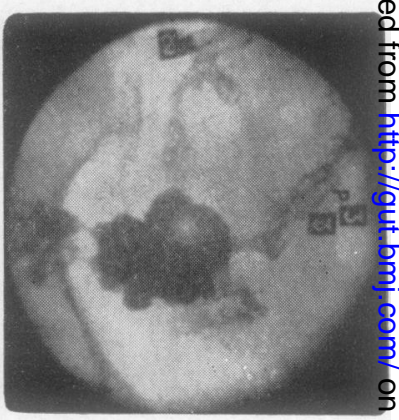

Frame 42

FIG. 1. The effect of morphine on the colon in diverticulosis (for details see text). $D=$ Diverticulosis, $L=$ Lead 2, $R=$ Rectum,$S=$ Sigmoid . 
reached heights of $20 \mathrm{~mm}$. $\mathrm{Hg}$ and $30 \mathrm{~mm} . \mathrm{Hg}$ respectively. As the neck of the diverticulum remained open during the entire film, it is obvious that the diverticulum must have been subject, at least in part, to the force of the intraluminal pressures that followed morphine.

Lead 2 recorded the pressure of $20 \mathrm{~mm}$. $\mathrm{Hg}$ when the bowel immediately above the diverticulum was narrowed (frame 10). This pressure was not registered by lead 1 , presumably because it was isolated from this zone of high pressure by the segmentation of the bowel. The tip of lead 1 cannot be seen in the film, being located in the mass of barium in the upper sigmoid. The diverticulum was less well-filled and the bowel above it less narrowed when the pressure fell to the basal level (frame 25), but it became better filled in frames 38 and 42 during a wave of pressure of $30 \mathrm{~mm}$. Hg. These frames also show the narrowing of the bowel above the tip of lead 2 that accompanied the rise in pressure which squeezed barium into the diverticulum, causing it to have a more definite outline. The barium within the diverticulum produced a bifid shadow as it encircled a faecolith.

This film demonstrates that a diverticulum may be subject to the intrasigmoid pressures that develop after morphine. This particular diverticulum was not markedly distended by these pressures but sometimes morphine causes dramatic changes in diverticula.
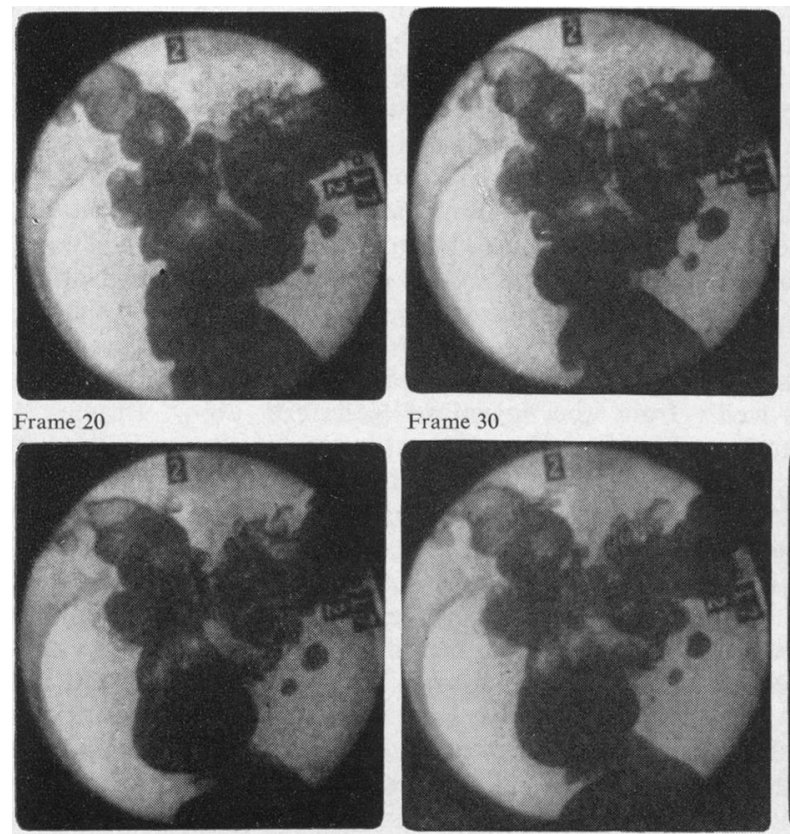

Frame 60 cula. Frame 30

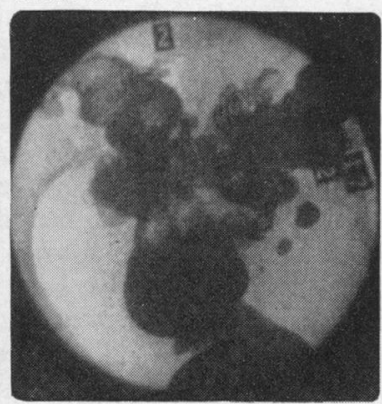

Figure 2 shows the sigmoid of a woman of 70. The sigmoid was so serpentine that the outline of its loops overlapped in the film and for this reason only the top recording lead could be passed beyond the recto-sigmoid junction into the lower sigmoid. Thus the intraluminal pressures near the two diverticula that are seen in the right lower quadrant of the prints could not be measured and so the pressure tracing is not reproduced. However, pressures of up to $20 \mathrm{~mm}$. Hg were recorded from the bowel below the diverticula after the intravenous injection of morphine. This injection was completed by the 21 st frame.

The necks connecting these two diverticula to the lumen of the colon are clearly seen in frame 20 . The intravenous morphine sulphate took effect rapidly and the bowel began to narrow in the next 10 seconds. Simultaneously the diverticula enlarged (frame 30) and their necks narrowed until at times they were shut completely (frame 50). The bowel from which they arose contracted so that its lumen was almost occluded (frame 60). When this film was projected, the movements of the colon were seen to be greatly increased and the diverticula appeared to be blown up like balloons. Although the necks of the diverticula were completely closed at times (frame 95), they opened intermittently (frame 99) and allowed barium to be pumped into the diverti-

This effect of morphine was not one that only

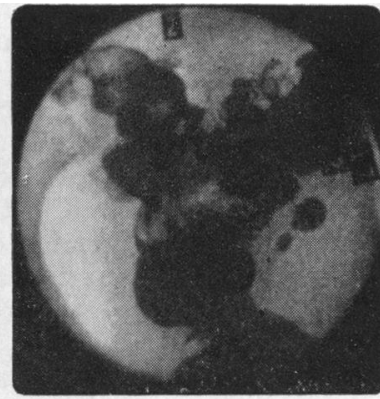

FIG. 2. The effect of morphine on colonic diverticula Frame 50 ( for details

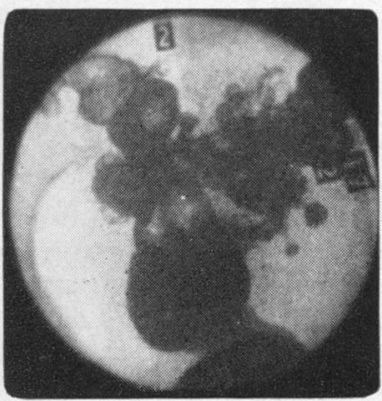
see text). 


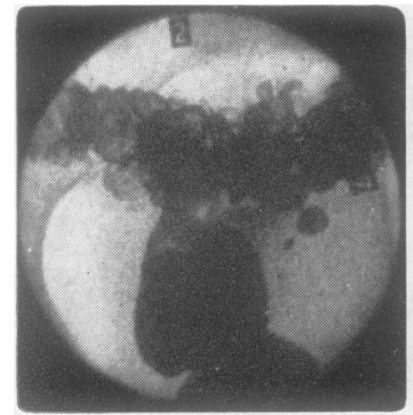

Frame 1

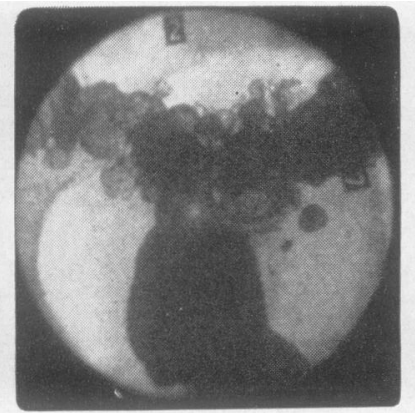

Frame 20

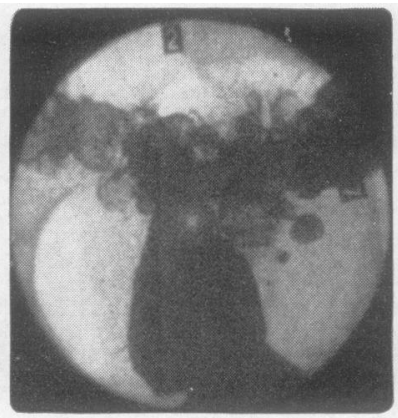

Frame 30

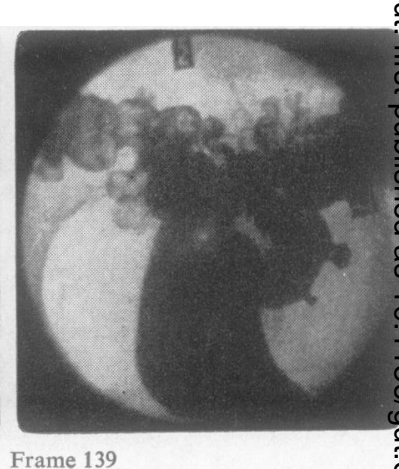

Frame 139

FIG. 3. The effect of probanthine on colonic diverticula that are under the influence of morphine (for details see text).

occurred immediately after its injection by the intravenous route as we have also observed it after intramuscular administration. Neither is it a shortlived response as a later film of the same subject will show (Fig. 3). This film was taken $\mathbf{4 0}$ minutes after the injection of morphine while $30 \mathrm{mg}$. of probanthine was being given intravenously. The diverticula were still distended (frame 1) before the probanthine took effect. The injection of this drug was completed by the eleventh frame. The colon was still active during these 11 frames but, during the remaining 130 frames, the motor activity of the bowel ceased, while the pressure tracing from the lower sigmoid became flat. The colon appeared to dilate as it became immobile (compare frames 20 and 139), and as the sigmoid relaxed, the necks of the diverticula opened (frames 20 to 30). The diverticula then diminished in size as their contents drained into the bowel (frame 139).

Prostigmine has an effect on the intraluminal pressures similar to that of morphine. Cineradiography reveals that prostigmine causes the interhaustral folds to contract in much the same manner as does morphine so that the colon has the segmented appearance which is usual when it is generating high localized intraluminal pressures. This effect of prostigmine is seen in health as well as in diverticulosis. Like morphine, prostigmine causes colonic diverticula to be subjected to high pressures (Painter et al., 1964), but while this effect is of practical importance in the case of morphine, as far as prostigmine is concerned this effect is only of theoretical interest at the present time.

Pethidine does not cause the sigmoid to produce an increased number of pressure waves either in health or in diverticulosis; in fact the number and dimensions of these waves are slightly diminished. It appears that the drug achieves this effect by depressing the excitability of the sigmoid so that it does not segment in response to stimuli that other- wise cause it to do so. Figure 4 shows the reaction of the sigmoid colon of a woman of 64 with diverticulosis to an injection of barium into its lumen. Three polythene tubes were located in the sigmoid and their tips can be seen in frame 5. Leads 1 and 2 recorded pressure while a third lead, the 'feeder' tube or lead F, was placed between them to facilitate the injection of barium into the sigmoid without interrupting pressure recording by the other two leads. Twenty $\mathrm{ml}$. of barium was injected via lead $\mathrm{F}$ between the second and twelfth frames. This barium passed up the sigmoid. The bowel around lead 1 responded by segmenting and generating a wave of pressure that reached a height of $19 \mathrm{~mm}$. $\mathrm{Hg}$ (frames 13 and 18). Diverticula appeared near lead 1 as this pressure built-up and can be seen on the right-hand side of the bowel. A second injection of barium was made between the 26th and 33rd frames; most of this flowed back to the rectum, being apparently unable to pass upwards as the upper sigmoid was now narrowed by the segmentation that had occurred (frames 23, 30, and 40). The bowel reacted to this second injection by again segmenting.

Figure 5 shows the same sigmoid after pethidine; the tips of the recording leads can be recognized through the shadow cast by the barium remaining from the injections described above (frame 3). Twenty $\mathrm{ml}$. of barium was injected via lead $F$ between the third and tenth frames and it was seen to pass along the sigmoid like water flowing through a drain (frames 5,10 , and 65 ). The barium passed through the open lumen of the sigmoid without being hindered by the configuration of the colonic wall for the lumen did not narrow and no contraction rings formed. In short, the colon did not segment. As we have come to expect, this lack of segmentation was accompanied by an absence of high intraluminal pressure. The diverticula now appeared as squat projections with widely open necks and are quite unlike their appearances seen 


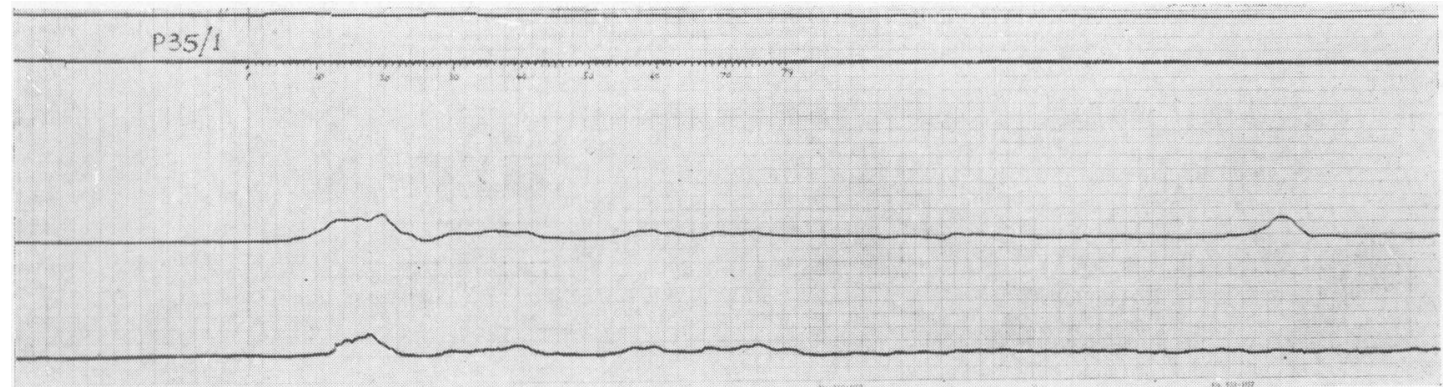

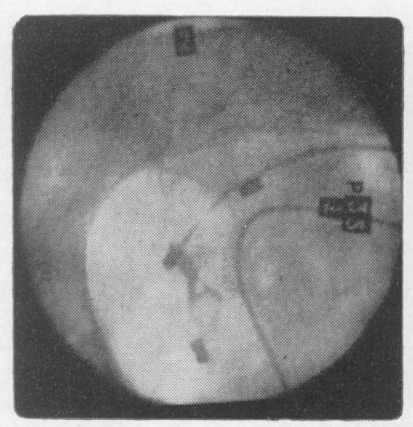

Frame 5

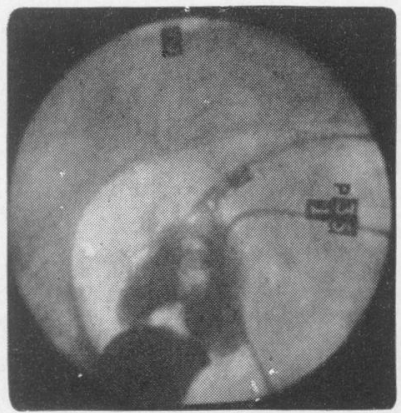

Frame 23

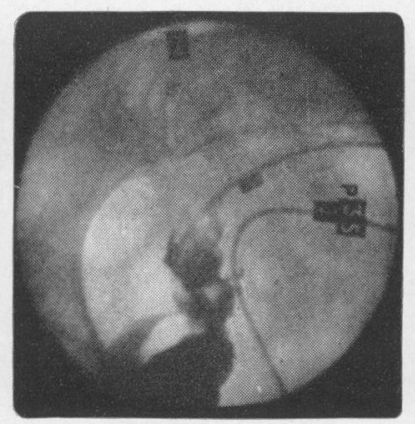

Frame 13

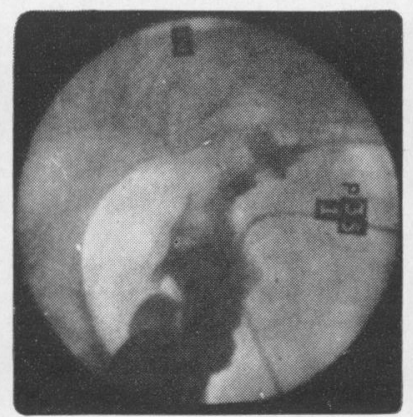

Frame 30

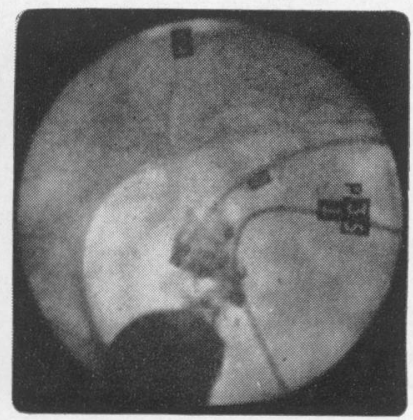

Frame 18

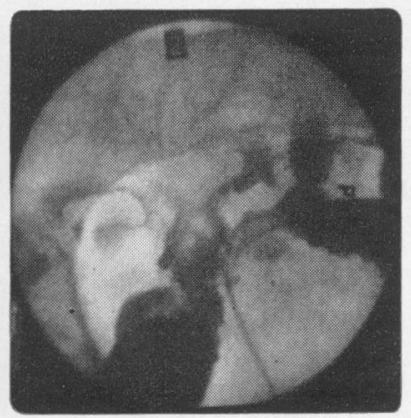

Frame 40

FIG. 4. The effect of an injection of barium into the colon in diverticulosis (for details see text).

in the previous figure. Clearly pethidine had affected the sigmoid colon so that it hardly responded to a stimulus that had previously excited it. The appearances of the colon and the diverticula after pethidine have some of the features that are seen after the administration of probanthine.

\section{DISCUSSION}

Cineradiography has shown conclusively that colonic diverticula are exposed to the force of the intraluminal pressures evoked by morphine. Once it is realized that administration of this drug is followed by the generation of intraluminal pressures that may exceed $90 \mathrm{~mm}$. $\mathrm{Hg}$ in those segments of the sigmoid colon that bear diverticula, the wisdom of giving it to patients with acute diverticulitis must be questioned.

In the early stages of diverticulosis, morphine causes the colon to segment and thus facilitate the generation of localized high intrasigmoid pressures while diverticula remain in communication with the lumen of the bowel. This stage of affairs favours the further herniation of the mucosa or the actual rupture of the diverticula, especially if they are inflamed. In some patients, the necks of the diverticula may be intermittently narrowed or completely closed after the administration of morphine. The degree of protection thus afforded to the diverticula is problematical. Whether the constricted necks act 


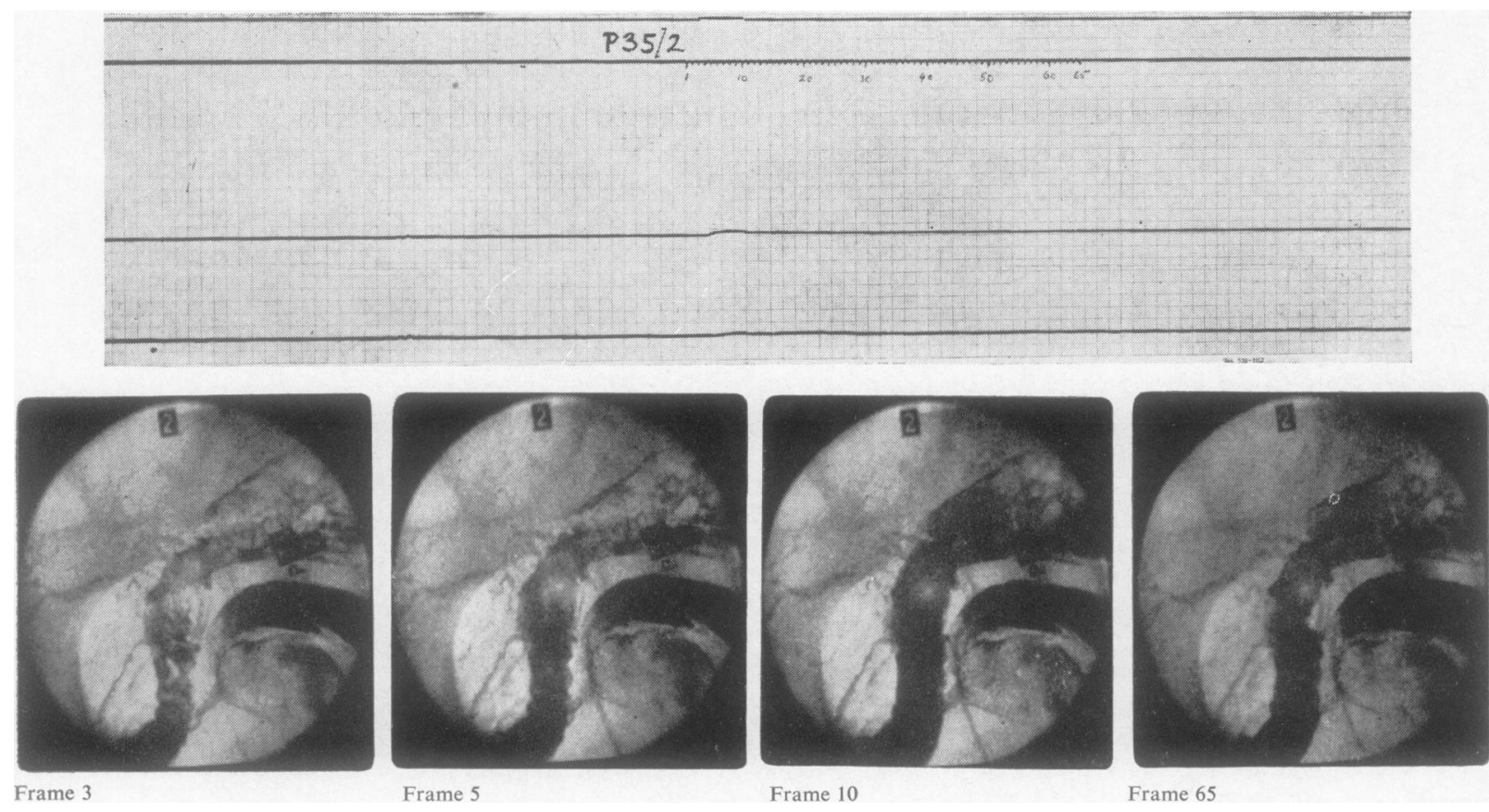

FIG. 5. The effect of an injection of barium into the lumen of the colon in diverticulosis after the administration of pethidine (for details see text).

as valves so that tension builds up within the diverticula, or whether the smaller channels between them and the bowel lessen the force of the intraluminal pressure that reaches the herniations, is uncertain. However, this much is certain; the occlusion of the necks after morphine is intermittent and they open at intervals sufficiently to allow colonic contents to be squirted into the ill-supported sacs of mucous membrane so that they are inflated like balloons, sometimes to an alarming extent.

In diverticulosis of long standing, the bowel may be so damaged by recurrent diverticulitis that parts of the muscle coat are unable to contract through being transformed into rigid fibrous tissue. Diverticula arising from such a sigmoid are not afforded any protection as their necks are widely open after morphine while high intraluminal pressures are occurring in their vicinity.

These observations suggest that morphine may be responsible for some of the perforations that occur when acute diverticulitis is treated expectantly. One of us (N.S.P.) has operated on 23 cases of perforated diverticulitis; three of these had no evidence of perforation on admission to hospital. All three patients were given morphine before perforation occurred; in two of them perforation did not occur until they had been in hospital for 48 hours. In retrospect, we wonder whether the drug was responsible for these catastrophes.

Furthermore, although the colon may be immobile at times after morphine, when bouts of activity do occur, its contractions are increased by the drug. It is obvious that this response cannot be beneficial in the inflamed colon of acute diverticulitis nor can it be desirable in other inflammatory diseases of the pelvis. What effect the post-operative use of morphine has on anastomoses situated in the sigmoid remains to be discovered. Restorative resections of the rectum are followed by some leakage of faeces at the site of anastomosis in up to $25 \%$ of patients, according to some authorities. While the poor blood supply of this part of the bowel does not facilitate the healing of these anastomoses, it is also possible that morphine encourages their disruption.

These adverse side-effects of morphine can be counteracted by giving probanthine simultaneously. This rests the bowel by paralysing it, prevents the generation of high pressures, and allows the diverticula to drain by opening their necks. It must be remembered that this drug in the doses we have used may cause paralytic ileus, so that its dosage and its place in the treatment of acute diverticulitis remain to be evaluated. Fortunately there is no 
need to use the combination of morphine and probanthine as pethidine is readily available.

Pethidine almost abolishes the generation of waves of high pressure in the sigmoid, both in health and in diverticulosis. It achieves this by lessening the tendency of the colon to segment in response to various stimuli, so that the lumen of the sigmoid remains open for longer periods than normally. The sigmoid is therefore less able to produce zones of localized high pressure. Furthermore, pethidine has a somewhat similar action to probanthine in that it allows diverticula to drain into the bowel as their necks remain open after its administration.

Prostigmine has an action that is similar to that of morphine as far as the intrasigmoid pressures and segmentation are concerned, both in health and in diverticulosis. Diverticula are also subject to the pressures that it evokes. While this finding is of no clinical importance, it does suggest that other drugs which cause segmentation may be followed by high intraluminal pressures that are transmitted to the diverticula. Localized high pressures in the sigmoid colon, being generated by the same mechanism that was originally responsible for the mucosal herniation, are almost certainly transmitted to diverticula regardless of the stimulus or drug that evokes them.

In brief, the use of morphine in acute diverticulitis appears to be contraindicated and pethidine is a preferable analgesic in this disease.

\section{REFERENCES}

Painter, N. S. (1962). Diverticulosis of the colon. M.S. Thesis, University of London, 1962.

-, and Truelove, S. C. (1964a). The intraluminal pressure patterns in diverticulosis of the colon. Part II. The effect of morphine. Gut, 5, 207-213.

(1964b). The intraluminal pressure patterns in diverticulosis of the colon. Part III. The effect of prostigmine. Gut, 5, 365-369. (1964c). The intraluminal pressure patterns in diverticulosis of the colon. Part IV. The effect of pethidine and probanthine. Gut, 5, 369-373.

, Ardran, G. M., and Tuckey, M. (1964). Segmentation and localization of intraluminal pressures in the human colon, with special reference to the pathogenesis of colonic diverticula. In preparation. 